\title{
س
}

$>\mathrm{DE}$

름AGOGIE

\author{
Recherches en éducation
}

$181 \mid 2012$

Varia

\section{Apprendre à faire classe. Les apprentissages professionnels des professeurs des écoles en formation par alternance}

Learning to delivers classes. Professional learning of primary school teachers in work-study programmes

Marc Daguzon et Roland Goigoux

\section{OpenEdition}

Journals

Édition électronique

URL : http://journals.openedition.org/rfp/3898

DOI : $10.4000 /$ rfp.3898

ISSN : 2105-2913

Éditeur

ENS Éditions

Édition imprimée

Date de publication : 31 décembre 2012

Pagination : 27-42

ISBN : 978-2-84788-405-0

ISSN : 0556-7807

\section{Référence électronique}

Marc Daguzon et Roland Goigoux, «Apprendre à faire classe. Les apprentissages professionnels des professeurs des écoles en formation par alternance », Revue française de pédagogie [En ligne], 181 | 2012, mis en ligne le 17 juin 2015, consulté le 26 juillet 2020. URL : http://journals.openedition.org/ rfp/3898; DOI : https://doi.org/10.4000/rfp.3898 


\section{Apprendre à faire classe. Les apprentissages professionnels des professeurs des écoles en formation par alternance}

\section{Marc Daguzon et Roland Goigoux}

Comment les professeurs des écoles apprennent-ils leur métier dans une formation en alternance ? L'étude longitudinale des apprentissages professionnels d'une quinzaine d'entre eux montre qu'ils attribuent tout d'abord un rôle prescriptif et normatif à leur formation, ce qui leur permet de définir les contours structurants d'un idéal professionnel. Dans un deuxième temps, confrontés à l'exigence de "faire classe » en pleine responsabilité, ils entreprennent un processus différencié de redéfinition de leur propre tâche d'enseignement qui interroge l'idéal régulateur. La réussite de leurs premières expériences pratiques et l'ajustement des conseils dont ils bénéficient semblent conditionner leur développement professionnel.

Mots-clés (TESE) : formation initiale des enseignants, établissement de formation des enseignants, formation en alternance, formateur.

\section{INTRODUCTION : LA FORMATION EN ALTERNANCE}

Dans la France de 2012, la formation initiale des professeurs des écoles dure trois ans: deux sous le statut d'étudiant - en master - pour maîtriser les savoirs à transmettre, acquérir les premiers éléments de pratique professionnelle et préparer les concours de recrutement, puis une troisième année en alternance, en cas de réussite aux concours, sous le statut de professeur des écoles stagiaire. Ce nouveau dispositif de formation, initié en 2010, est trop récent pour avoir été évalué. Le précédent, qui ne comportait qu'une année préparatoire avant l'entrée en fonction, avait en revanche été étudié par plusieurs équipes de recherche qui avaient identifié quelques-uns des invariants de l'entrée dans le métier (voir par exemple la synthèse de Maulini, 2009). Mais les deux dispositifs ont un point commun : une année de formation en alternance au cours de laquelle les stagiaires partagent leur temps, selon des proportions variables, entre des stages de pratique accompagnée réalisés dans les classes de maîtres chevronnés, des stages en pleine responsabilité, seuls face à une classe, et des enseignements dispensés à l'Institut universitaire de formation des maîtres (IUFM). C'est cette année de formation en alternance qui est l'objet de la recherche présentée dans cet article.

En 2006, un important colloque a permis de construire une image précise des dispositifs et scénarios de formation effectivement mis en œuvre dans les IUFM 
(Robert, Goigoux, Brigaudiot et al., 2006). La diversité des modalités d'organisation de l'alternance entre les stages et les cours dispensés dans les instituts de formation y a été décrite (Goigoux \& Paries, 2006). Les principaux contrastes tenaient aux principes qui soustendaient ces organisations : si certaines équipes de formateurs tentaient de s'approcher de la globalité de l'acte d'enseignement, les autres revendiquaient le caractère nécessairement analytique de la formation. Les unes privilégiaient une formation au plus près du terrain, celui-ci étant vu comme un moyen privilégié pour favoriser l'appréhension simultanée de toutes les composantes du métier puisque, par définition, la pratique effective les convoque toutes. Les autres revendiquaient une approche plus fragmentaire et plus réductrice que seul un institut de formation, préservé momentanément des urgences de l'action, peut envisager. Aucun scénario de formation n'échappait à la mutilation d'une partie de l'activité professionnelle, du fait même de sa conceptualisation dans le cadre de la formation extérieure au contexte de l'action : il ne s'agissait pas d'une mutilation subie mais bien d'un choix délibéré qui consistait à réduire la complexité de l'activité d'enseignement pour en faciliter l'apprentissage. Bref, si chacun essayait de prendre en compte la globalité et la complexité de l'action, les moyens pour y parvenir étaient différents, tant sur le plan des dimensions de l'activité enseignante qui étaient isolées que sur le plan de la recomposition et de la recontextualisation de ces dimensions.

En 2008, un second colloque faisant suite au premier a été consacré à l'analyse des parcours de formation des enseignants débutants (Goigoux, Ria \& Toczek, 2009a). Il a permis de mieux comprendre ce que pensaient les stagiaires des dispositifs de formation dont ils bénéficiaient (voir aussi Wittorski, 2007 ; Wittorski \& Briquet-Duhaze, 2008). Dans leur synthèse, Goigoux, Ria et Toczek (2009b) notent que bon nombre de stagiaires déplorent l'écart qui sépare "la théorie » de "la pratique " même si tous ne donnent pas le même sens à ces deux mots. Le premier problème qu'ils soulèvent est celui d'apports jugés trop théoriques parce qu'ils ne constituent pas des ressources pour l'action professionnelle ou qu'ils exigent de trop longues et trop complexes transpositions personnelles. Dans ce cas, l'adjectif "théorique " ne s'oppose plus à " pratique ", mais est synonyme d'utopique, c'est-à-dire quelque chose que le débutant perçoit comme étant au-delà de ses compétences et qu'il est donc incapable de réaliser. Ici la " théorie » est donc une pratique jugée peu réaliste en raison de sa complexité : ce qui était faisable en formation, dans des conditions privilégiées, ne l'est plus au quotidien, sur le terrain scolaire ordinaire. Alors que dans le centre de formation le stagiaire avait beaucoup de temps pour préparer une seule séquence didactique, qu'il pouvait en discuter avec ses collègues et ses formateurs, qu'il disposait de ressources documentaires, il se sent démuni lorsqu'il se retrouve seul, pris par l'urgence et confronté à une multiplicité de tâches.

Le manque de réalisme exprimé par les stagiaires interrogés découle aussi du choix de certains formateurs qui proposent aux débutants des scénarios innovants et, par conséquent, rares : seuls quelques professeurs très expérimentés, en lien avec des associations de spécialistes, des mouvements pédagogiques ou des centres de formation, les mettent en œuvre sur le terrain. Du coup, les débutants ont peu de chance de trouver un collègue, dans une classe voisine de la leur, avec lequel partager leur expérience. Aussi la plupart d'entre eux abandonnent-ils très vite ces pratiques, bien qu'ils en reconnaissent le bienfondé, s'ils ne sont pas solidement épaulés (Butlen, Peltier-Barbier \& Pézard, 2002). Ils jugent qu'elles sont prématurées et relèvent de la formation continue. L'écart entre « théorie » et " pratique » est, dans ce cas, un écart temporel : "Ce que vous nous proposez est intéressant, disent les néo-titulaires à leurs formateurs, mais cela n'arrive pas au bon moment, c'est trop tôt dans notre carrière, nous avons d'autres problèmes à régler. 》

D'autres raisons encore sont invoquées pour juger inadéquates certaines recommandations des instituts de formation. Certains débutants déplorent qu'elles émanent d'enseignants (maîtres formateurs par exemple) exerçant dans des conditions privilégiées par comparaison à celles de leurs débuts de carrière : classes bourgeoises des centres-villes versus quartiers populaires, équipes pédagogiques versus isolement, etc. Ils disent aussi parfois qu'elles sont formulées par des formateurs permanents trop éloignés du terrain, c'est-à-dire selon eux trop ignorants des réalités et des contraintes qui pèsent sur l'exercice du métier. C'est le cas, par exemple, quand des formateurs survalorisent la dimension didactique de l'activité d'enseignement au détriment des autres, quand ils considèrent que les décisions à prendre en classe découlent exclusivement de l'examen rationnel des notions à enseigner. Or les débutants savent que lorsqu'ils sont en classe, ils prennent quotidiennement de multiples décisions dans bien d'autres buts que de favoriser les apprentissages des élèves : pour obtenir le calme, pour capter l'attention des élèves, pour préserver l'affection que ceux-ci leur portent, pour 
entretenir leur propre motivation ou pour économiser leurs forces, etc. (Peltier-Barbier, 2004).

À la recherche de solutions fonctionnelles, voire de stratégies de survie, les débutants interrogés disent recourir à des pratiques jugées régressives par l'orthodoxie didactique ou pédagogique. Insatisfaits, ils contestent les reproches qui leur sont adressés. Ils ne remettent pas en cause toute théorisation mais dénoncent une formation culpabilisante n'offrant pas suffisamment de traductions concrètes des théories enseignées. Ils rejoignent en cela ceux qui, moins démunis cependant, vantent la " richesse théorique » de la formation en IUFM, «quelque chose vers quoi il faut tendre » et qui sert de base à leur action pédagogique. Dans tous les cas, les débutants s'efforcent de trouver en classe un bien-être suffisant pour « tenir » jour après jour. Ils semblent en quête d'un équilibre entre deux logiques de planification et de régulation de leur activité : la logique des savoirs enseignés (la logique didactique stricto sensu) et la logique de la conduite de la classe (celle de la régulation sociale des échanges et des comportements). Ils découvrent vite que la seconde peut prendre le pas sur la première lorsque les conditions d'exercice se dégradent : plus le nombre d'élèves en difficulté augmente, plus leurs décisions visent avant tout à maintenir "la classe en vie ", c'est-à-dire en ordre et en activité, choix qui se fait parfois au détriment de la qualité des apprentissages. Tous souhaitent que les formateurs les aident à trouver comment réduire les tensions entre ces logiques souvent divergentes, bref à analyser et à comprendre les compromis qui peuvent assurer la cohérence de leur pratique. Or, loin de tout compromis, certains formateurs proposent un idéal qui, s'il sert de boussole aux uns, désespère les autres qui retournent leur colère, née d'un sentiment d'impuissance, vers l'institut de formation (Daguzon, 2009).

\section{PROBLÉMATIQUE}

La recherche que nous avons réalisée dans le cadre des dispositifs de formation antérieurs à la réforme de 2010 est née de ces constats que notre propre expérience de formateurs nous avait permis d'approcher. Nous nous interrogions sur l'adéquation des scénarios de formation aux compétences des stagiaires et néo-titulaires. Nous faisions l'hypothèse que les formateurs ne connaissaient pas assez bien la zone proximale de développement professionnel des débutants pour y intervenir efficacement et pour différencier leurs interventions à la hauteur de l'hétérogénéité de leur public (Altet, 1994).

Les publications de recherches dont nous disposions apportaient des éclairages sur certaines caractéristiques des apprentissages des enseignants débutants (Huberman, 1989 ; Baillauquès, 1999 ; Tardif \& Lessard, 1999), sur les dynamiques de leur développement professionnel (Piot, 1997 ; Legendre, 1998) et sur leurs transformations identitaires (au sens de transformations des " conceptions de soi au travail »; Dubar, 2002 ; Wittorski, 2007). Elles indiquaient par exemple que les débutants sont avant tout préoccupés par le fait de «tenir leur classe » ou de "faire autorité » et que la reconnaissance qui en découle provient à la fois des élèves, des collègues et des parents d'élèves : cette compétence fonde le sentiment de professionnalisme qui se forge à l'occasion des premières expériences de responsabilité de classe (Lang, 1999). Mais elle se heurte très vite aux tensions, voire aux contradictions dans la conduite de la classe, entre les logiques d'apprentissage d'une part et les logiques de socialisation d'autre part. L'équipe de Denis Butlen (2004) les explique de la manière suivante : Iorsqu'ils enseignent dans des conditions difficiles, les débutants sont avant tout préoccupés par l'installation d'une "paix scolaire ", combinaison d'une « paix sociale » et d'une « adhésion au projet d'enseignement du professeur ». II s'agit d'établir et de faire accepter par les élèves des règles de fonctionnement permettant d'obtenir le calme, le contrôle des prises de paroles et un climat de sécurité, tout en parvenant à les enrôler rapidement et sans trop de résistance dans les tâches scolaires. Pour y parvenir, ils recourent à un certain nombre de gestes professionnels que les chercheurs ont inventoriés : exercer une "pression " constante sur les élèves, maintenir un rythme de travail soutenu, remporter l'adhésion de tous les élèves en ménageant une place à chacun, garder le contact avec eux en restant attentif à leurs questions, etc. On observe que cette paix scolaire est le plus souvent obtenue par l'imposition d'une certaine " discipline ». Mais rares sont les débutants qui, lorsqu'ils exercent en milieux difficiles, obtiennent l'adhésion des élèves à leur projet d'enseignement sans réviser leurs ambitions à la baisse ou raccourcir les temps d'activité.

Des recherches complémentaires ont montré que lorsqu'ils exercent dans des situations moins difficiles, les professeurs débutants s'efforcent progressivement de satisfaire d'autres exigences et d'affronter d'autres problèmes (Kagan, 1992). Ils cherchent à avancer 
dans le programme et à enchaîner les séquences didactiques en se basant sur ce que la majorité des élèves semble capable de suivre. Ils veulent mettre les élèves au travail et, si possible, " en activité ", c'est-à-dire dépasser la simple occupation pour atteindre une activité constructive du point de vue des apprentissages. Les critères retenus pour évaluer la réussite de cette entreprise sont la participation des élèves aux échanges en classe et le maintien de leur attention tout au long des séances didactiques. Ce n'est que plus tard, lorsqu'ils ont réglé les principaux problèmes de conduite de classe, que les débutants parviennent à vérifier si l'enseignement dispensé débouche bien sur les apprentissages visés, voire à différencier leur enseignement. Ils s'efforcent alors de concilier l'avancée du temps didactique avec le respect de la parole enfantine, de motiver les élèves sans les détourner des enjeux des apprentissages, de conduire la classe sans se fixer sur un niveau moyen qui exclut les plus faibles, de favoriser l'activité des élèves sans dénaturer les objets de savoir (Goigoux, 2006).

À l'issue de cette revue de questions, il apparaissait que de nombreuses interrogations restaient sans réponse, en particulier sur la stabilité et l'invariance des descriptions réalisées (Paquay, Altet, Charlier et al., 2012) : peut-on parler d'un modèle du développement professionnel relativement commun, composé d'étapes successives ? Tous les débutants ont-ils besoin de maîtriser la conduite de leur groupe classe avant de pouvoir prendre en compte les différences interindividuelles ou le mouvement développemental peut-il aller dans le sens inverse ? C'est pour répondre à ces questions que nous avons entrepris notre recherche, d'autant plus nécessaire que l'expérience de la responsabilité d'une classe est ressentie comme une épreuve, au double sens de rite initiatique et de souffrance, accompagnée parfois d'une angoisse de la non-validation (Blanchard-Laville \& Nadot, 2000).

Dans l'alternance, l'épreuve de la prise de responsabilité ne semble pouvoir contribuer à un développement professionnel harmonieux que si elle est franchie avec succès, ce qui implique que les conseils apportés lors des stages et les enseignements délivrés dans le centre de formation soient ajustés aux compétences présentes chez tous les stagiaires. Faute de quoi ils peuvent être jugés passionnants par les uns et inutiles par les autres. Dans cette perspective, nous faisons l'hypothèse que la formation peut produire des effets très différents selon le niveau initial des enseignants débutants. Pour tester cette hypothèse, nous avons donc étudié comment les professeurs des écoles débutants (ci-après PE2, professeurs des écoles $2^{\mathrm{e}}$ année) apprennent à faire classe durant leur année de formation en alternance. Nous voulions en particulier comprendre comment les savoirs théoriques et les savoirs pratiques peuvent s'articuler et se compléter (Pastré, 2008) au cours de cette alternance.

Pour cela, nous avons choisi d'analyser :

- ce que les professeurs débutants s'efforcent de faire lorsqu'ils sont en classe (stages en responsabilité) ;

- ce qui les préoccupe pendant la préparation et la réalisation de leur enseignement ;

- comment évoluent les buts qu'ils se donnent et les conceptions pédagogiques qui sous-tendent leurs pratiques de classe au cours de cette année de formation professionnelle ;

- ce qui influence cette évolution, notamment les prescriptions formulées par l'employeur (ministère de l'Éducation nationale) et par le centre de formation.

\section{CADRE THÉORIQUE}

Notre recherche visait à cerner les buts que les enseignants débutants se donnent à eux-mêmes, ce que Jacques Leplat décrit comme « l'élaboration par le sujet de sa propre tâche » (1997, p. 16) et qu'il appelle la " tâche redéfinie ». Bien que celle-ci puisse être inférée à partir d'éléments observables de l'activité, nous avons privilégié l'étude de la parole des débutants en nous référant au cadre théorique proposé par Leplat dans le champ de la psychologie ergonomique (1992, 1997 ; voir aussi Rogalski \& Leplat, 2011), complété par celui la didactique professionnelle (Pastré, 2005, 2011) et de la didactique des disciplines (Goigoux, 2007). Le concept de redéfinition de la tâche nous a permis d'examiner comment les professeurs des écoles débutants donnaient un sens à leur activité et évaluaient la réussite de leur action.

Pour comprendre le passage du travail prescrit au travail réel, il nous a fallu étudier comment les PE2 traduisaient les prescriptions en intentions d'action et comment ils poursuivaient leurs propres fins en même temps que celles assignées par les tâches prescrites. Le plus pertinent était d'étudier cette redéfinition au moment où elle se construisait, c'est-à-dire au moyen d'une étude longitudinale révélant l'évolution des représentations fonctionnelles (Leplat, 1985 ; Pastré, 2005) au fur et à mesure de l'avancée des cours 
dispensés à l'IUFM et des expériences réalisées en stage. Nous avons considéré que, durant leur année de formation en alternance, nous pouvions saisir un processus d'actualisation des conceptions des débutants qui s'opérationnalisaient et se diversifiaient, se transformant en des représentations fonctionnelles pour assurer la planification et le guidage de l'action durant les stages en responsabilité. Leplat et Hoc (1983) ont introduit la notion de représentation fonctionnelle comme une hypothèse de recherche pour rendre compte de différentes situations de travail où la prédiction est un but de l'activité. S'appuyant sur la notion d'image opérative (Ochanine, 1978), ils associent la représentation fonctionnelle à un modèle intériorisé de l'action qui n'a de sens que dans le rapport qu'il entretient avec l'exécution de la tâche. Ils repèrent deux propriétés qui confirment la finalité prédictive de la représentation fonctionnelle : d'une part, elle est sélective dans la mesure où elle ne retient que les éléments pertinents pour l'action en contexte et, d'autre part, elle est déformante puisque ces éléments seront perçus avec plus d'acuité par l'opérateur que par un observateur extérieur non engagé dans la tâche (Ochanine, 1978). En ce sens, les représentations fonctionnelles sont des construits de l'activité cognitive de l'acteur dans le but de réaliser la tâche prescrite.

Pour identifier ces représentations, nous avons étudié les préoccupations de stagiaires, notamment lorsqu'elles manifestaient des tensions ou des préoccupations concurrentes. Nous savions que, dans la pratique, les débutants sont souvent placés face à des dilemmes (Butlen, Peltier-Barbier \& Pézard, 2002) : socialisation des élèves versus apprentissages, réussite versus conceptualisation, individu versus collectif, temps de la classe versus temps des apprentissages, etc. Selon nous, ceux-ci ont pour origine la multifinalité de l'activité enseignante qui engendre nécessairement des conflits de critères (Carter \& Doyle, 1987). C'est l'étude de ces conflits qui a été au centre de nos analyses puisque nous avons systématiquement cherché à identifier les dimensions potentiellement antagonistes de cette activité et la manière dont les débutants parvenaient à réduire les tensions entre des logiques parfois divergentes, bref à analyser et à comprendre les compromis qui assuraient la cohérence de leurs propos et de leurs actions.

Nous savions aussi que la résolution des dilemmes rencontrés en situation d'enseignement pouvait accélérer le développement professionnel des débutants, en fonction des conceptions dont ils étaient porteurs (Harrington, Quinn-Leering \& Hodson, 1996) et de la particularité des situations dans lesquelles les dilemmes émergeaient. Deux études (Freese, 1999 ; Tillema, 2000) montraient, en effet, que les débutants construisent bon nombre de leurs connaissances à la suite d'une réflexion consciente commencée pendant l'action. Celle-ci donne lieu à une prise de décision " à chaud " dont l'analyse a posteriori sert de base à la construction d'un nouveau savoir professionnel. Pour identifier les sources d'influence de l'évolution des représentations fonctionnelles, nous avons distingué la prescription primaire du travail, constituée par tout ce que l'institution scolaire définit et communique au professeur pour l'aider à concevoir, à organiser et à réaliser son travail et la prescription secondaire, émanant de I'IUFM. Cette dernière est élaborée et diffusée par les formateurs qui, non seulement reformulent, interprètent ou concrétisent les injonctions hiérarchiques, mais développent aussi un ensemble de recommandations autonomes (Goigoux, 2002). C'est par l'analyse de l'origine des prescriptions retenues par les débutants et par l'étude de leurs propres caractéristiques (expériences antérieures, formation initiale, systèmes de valeurs, etc.) que nous avons pu identifier les sources qui modifient leurs conceptions pédagogiques.

\section{MÉTHODOLOGIE}

Nous avons réalisé une étude longitudinale portant sur quinze PE2 : de septembre à juin, ceux-ci ont effectué treize semaines de stage (un stage d'observation de deux semaines, un stage de pratique accompagnée de deux semaines, trois stages en responsabilité de trois semaines). Au cours de la même période, ils ont bénéficié de vingt-deux semaines de cours à l'IUFM d'Auvergne.

À intervalles réguliers, nous avons conduit des entretiens que nous avons enregistrés puis retranscrits. Nous voulions savoir ce que les stagiaires retenaient des prescriptions qui leur étaient adressées et comment évoluait, tout au long de leur année de formation professionnelle, ce qu'ils jugeaient bon de faire : "Qu'est-ce que vous croyez que l'on attend de vous ? Qu'en retenez-vous et que vous efforcezvous de faire en classe ? " Les entretiens étaient de trois types (entretien individuel semi-directif, entretien collectif et autoconfrontation individuelle à partir d'enregistrements vidéo de leur activité 
Tableau 1. L’opération Moleskine - Planning des différents entretiens

\begin{tabular}{|c|c|c|c|c|c|c|c|c|c|}
\hline Sept. & $\begin{array}{l}\text { Sept.- } \\
\text { Oct. }\end{array}$ & Oct. & $\begin{array}{l}\text { Oct.- } \\
\text { Nov. }\end{array}$ & $\begin{array}{l}\text { Nov.- } \\
\text { Déc. }\end{array}$ & $\begin{array}{l}\text { Déc. à } \\
\text { Mars }\end{array}$ & Mars & $\begin{array}{c}\text { Avril- } \\
\text { Mai }\end{array}$ & Mai & Juin \\
\hline 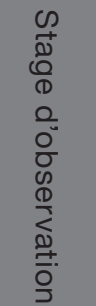 & $16 \mathrm{E}$ & 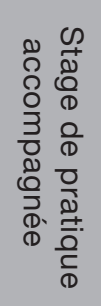 & $8 \mathrm{E}$ & 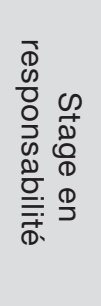 & $\begin{array}{l}15 \mathrm{E} \\
1 \mathrm{Co} \\
8 \mathrm{AC}\end{array}$ & 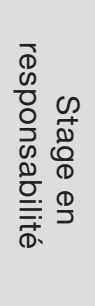 & $\begin{array}{l}8 \mathrm{E} \\
1 \mathrm{Co} \\
8 \mathrm{AC}\end{array}$ &  & $\begin{array}{c}3 \mathrm{E} \\
1 \mathrm{Co} \\
8 \mathrm{AC}\end{array}$ \\
\hline
\end{tabular}

Lecture : $E$ : entretien individuel semi-directif ; $A C$ : entretien individuel en autoconfrontation simple ; Co : entretien collectif.

d'enseignement) afin que leurs recoupements rendent plus fiable l'interprétation des données.

- 1er type (E) : huit entretiens individuels semi-directifs par stagiaire. Chacun des huit premiers PE2 rapportait, en une trentaine de minutes, les prescriptions qui lui avaient été adressées depuis l'entretien précédent. Il était invité à indiquer celles qu'il jugeait pertinentes et qu'il reprenait à son compte pour l'avenir. Entre deux entretiens, il avait noté dans un carnet de moleskine ce qu'on lui montrait ou ce qu'on lui disait qu'il était «bon de faire »;

$-2^{e}$ type $(A C)$ : trois entretiens individuels en autoconfrontation simple par stagiaire. Chaque période de stage en responsabilité était suivie par une autoconfrontation simple au cours de laquelle chaque stagiaire commentait à l'intention des chercheurs sa propre activité d'enseignement filmée lors d'une visite d'un de ses tuteurs. Au-delà de sa part observable, il dévoilait ce qu'il s'efforçait de faire pendant la séance, parfois sans y parvenir ;

- 3e type (Co) : quatre entretiens collectifs. Un groupe de sept autres stagiaires a été réuni à quatre reprises, à chaque retour de stage durant trois heures chaque fois. Ces PE2 avaient exactement les mêmes consignes de collecte. Chacun prenait appui sur son expérience de stage pour commenter les prescriptions qui lui semblaient déterminantes. Le groupe réagissait alors pour indiquer s'il partageait ou non l'analyse de leurs collègues.

Les entretiens retranscrits ont donné lieu à une analyse de contenu dont le détail est exposé dans la thèse de Marc Daguzon (2010). Pour catégoriser les énoncés révélant la représentation fonctionnelle de la tâche d'enseignement que les stagiaires se donnaient, nous nous sommes référés au modèle d'analyse de l'activité de l'enseignant proposé par Goigoux (2007). Pour mieux cerner ce que les PE2 s'efforçaient de faire, nous nous sommes intéressés aux dimensions et aux finalités multiples qu'ils évoquaient (Dubet, 2002). Nous avons donc catégorisé les énoncés recueillis en quatre ensembles selon les principales orientations mentionnées :

- vers la classe : ce que dit le PE2 sur la manière dont il s'efforce de conduire et de faire vivre la classe en tant que groupe social qui entretient avec lui des rapports dont les règles ne sont ni totalement données à l'avance, ni définitivement établies ; comment il s'efforce de maîtriser la trajectoire intellectuelle et relationnelle de la classe considérée comme une entité dont il veut rester maître ; ce qu'il dit d'un déroulement convenable de la journée de classe, comment il s'assure que les comportements des élèves respectent les règles sociales actualisées par son autorité ou que les prises de paroles sont régulées, etc. ;

- vers les élèves : ce que dit le PE2 des objectifs et des moyens retenus pour favoriser les apprentissages de ses élèves, dans différents registres cognitifs et sociaux (instruire et éduquer) ; ce qu'il dit de la manière dont les élèves apprennent ; comment il s'assure qu'ils acquièrent les compétences visées, qu'ils sont en activité sur les objets prévus, etc. ;

- vers l'enseignant lui-même : ce que dit le PE2 des choix qu'il effectue en fonction des coûts et des bénéfices qu'il peut retirer personnellement de son activité, en fonction de ses propres buts qu'il peut décliner en termes d'objectifs pédagogiques, de valeurs, de fierté professionnelle, d'estime de soi, de confort, de santé, 
d'intégration à son milieu de travail, de reconnaissance sociale, de déroulement de carrière, etc. ; ce qu'il dit de ses efforts pour construire une image de son action conforme à ce qu'il pense que doit être et doit faire un enseignant ;

- vers les autres acteurs de la scène scolaire : ce que dit le PE2 des efforts consentis pour rendre lisible et acceptable (voire pour valoriser) son action professionnelle à leurs yeux ; comment il essaye d'intégrer son activité à celle des autres : sa hiérarchie, les autres enseignants, les parents des élèves, etc.

\section{PRINCIPAUX RÉSULTATS}

Les analyses réalisées révèlent que la redéfinition de la tâche faite par le PE2 est le fruit de la confrontation de ses conceptions de début d'année et de l'événement que constitue son stage en responsabilité. En septembre, sa conception de la tâche d'enseignement dépend essentiellement de ce qu'il a retenu de la prescription secondaire, c'est-àdire celle qui est exposée dans les cours dispensés à l'IUFM ou lors des stages de l'année précédente (PE1) et en début d'année de PE2. Durant la période qui précède le premier stage en responsabilité, les PE2 adoptent des stratégies différentes. Certains n'évoquent pas l'épreuve de la pratique et considèrent qu'ils apprennent gratuitement : ils diffèrent le moment où ils devront se confronter aux élèves. D'autres, à l'inverse, sont à la recherche de tous les conseils qui pourraient les aider à prendre en charge leur classe à venir. D'autres encore s'attachent plus à souligner les contradictions entre ce qu'ils ont retenu de la « théorie » et ce qu'ils ont vu réellement mis en œuvre dans les classes au cours de leurs stages. Néanmoins, l'anticipation de la responsabilité de la classe les conduit tous, a minima, à distinguer une prescription maximale et exigeante (" ce qu'il est bon de faire ») qu'ils évoquent en parlant de " ce qu'il faut faire en théorie ", et une prescription minimale ("ce qu'il faut au moins faire »), moins exigeante et plus réaliste, qu'ils évoquent en parlant de «la pratique ». Cette distinction, floue en début d'année, préserve l'avenir et permet d'avoir un cadre de justification pour expliquer leurs éventuelles difficultés en stage. En résumé, le début du parcours des $\mathrm{PE} 2$ révèle que la première étape du développement professionnel est fortement influencée par la prescription secondaire.

\section{Première période : avant l'expérience de la responsabilité de la classe}

\section{Une conception de la tâche centrée sur les apprentissages scolaires}

Au mois de novembre, juste avant leur départ en stage en responsabilité, les PE2 ont des conceptions de la tâche d'enseignement assez similaires, essentiellement orientées vers la prise en compte des apprentissages des élèves. Ils ont compris que l'activité de l'enseignant consistait essentiellement à faire apprendre quelque chose aux élèves, ce qui explique qu'ils décrivent plus facilement l'activité des élèves que leur propre activité d'enseignement. Cette dernière, la plupart du temps, est inférée à partir de l'activité de l'élève qu'ils doivent promouvoir. Tout se passe comme si les connaissances acquises sur les processus d'apprentissages des élèves tenaient lieu de viatique pour pouvoir enseigner.

Ce dernier se constitue essentiellement sous l'influence de trois injonctions conformes au modèle socioconstructiviste dominant dans le centre de formation :

- l'activité : «il faut que les élèves soient actifs » est le premier commandement, celui qui revient le plus régulièrement dans les propos des PE2. L'activité recherchée est observable, les élèves doivent être visiblement engagés dans une tâche qui suscite de l'intérêt et dans laquelle ils s'investissent. Les PE2 sont sensibles aux marques de motivation et d'ennui qui sont des indicateurs de la pertinence de la tâche choisie. La question de la motivation des élèves est d'ailleurs une de leurs préoccupations essentielles dans les phases de préparation de la classe. Ils ont retenu qu'il est nécessaire d'“ habiller " les tâches proposées pour favoriser l'engagement et l'adhésion des élèves ;

- la confrontation verbale : « il faut que les élèves parlent " est le deuxième commandement que les PE2 restituent dans les entretiens. Ils ont aussi retenu qu'il est nécessaire de prendre en compte la parole de chaque élève singulier et que celui-ci doit pouvoir exprimer l'état de ses interrogations pour deux raisons : d'une part parce qu'il révèle ainsi ses représentations sur les objets de savoir dont l'émergence est une étape essentielle du processus d'apprentissage dans le cadre socioconstructiviste, et d'autre part parce que la confrontation interindividuelle est une formalisation, adaptée à la classe, du conflit sociocognitif qui leur semble être à l'origine de toute nouvelle connaissance ; 
- l'autonomie : "il faut développer l'autonomie des élèves » est le troisième commandement. Celle-ci est systématiquement convoquée pour préciser les modalités d'organisation des tâches scolaires. Pour s'assurer de l'engagement et de la motivation de tous, les PE2 pensent que les tâches doivent être ajustées au mieux à leurs capacités cognitives, l'indicateur d'un ajustement réussi étant alors l'autonomie dont chaque élève pourra faire preuve dans la réalisation des tâches proposées.

\section{Identité et posture professionnelle}

Les PE2 construisent une représentation de l'identité de l'enseignant à partir de cette conception de la tâche orientée vers les apprentissages des élèves. Ils élaborent un modèle du " bon " enseignant en référence aux pratiques des maîtres formateurs qu'ils ont observées. Dans leur modèle, l'enseignant est avant tout un " concepteur ". II bâtit ses progressions et ses répartitions, élabore les différents types de leçons qui rythment les séquences d'enseignement, s'assure d'un découpage en séances équilibrées. II construit aussi les différents exercices et les évaluations qui lui permettront d'analyser son travail. Les PE2 se représentent donc l'enseignant comme étant d'abord un planificateur, fonction qu'il exerce en l'absence des élèves.

En classe, ils imaginent un enseignant en retrait, posture propre à un accompagnateur qui soutient l'activité autonome d'élèves engagés dans des tâches appropriées. Quitte à miser sur les autres élèves pour compenser ce retrait volontaire comme l'explique l'un d'entre eux : « En fait je me dis que, dans l'idéal par rapport à ce que je pourrais connaître de ce que l'on m'a dit à l'IUFM, si un enfant ne répond pas à ce que je pouvais attendre, je me dirais, idéalement il devrait y avoir un enfant qui serait capable de le contredire et de lui montrer avec ses mots, de le ramener dans le bon chemin... »

\section{La gestion de la classe}

La gestion de la classe est la grande absente de la représentation initiale des PE2, comme si l'attention portée aux apprentissages des élèves suffisait. À cette période de l'année, ils pensent que l'organisation temporelle, spatiale et matérielle doit être la mise en application de ce qui a été décidé au niveau de la préparation et que le déroulement de la journée est la résultante de cette organisation. Ils jugent que si le travail d'anticipation a été bien mené alors il n'y aura pas ou très peu d'écarts entre le prévu et le réalisé.
La relation d'autorité n'est pas problématisée et aucune prescription explicite sur la gestion des relations entre les élèves n'apparaît dans les premiers entretiens. Toutefois, on note que les PE2 refusent les postures directives parce qu'elles ne leur semblent pas adaptées au type de relation qui découle de la mise en activité des élèves. Pour autant, ils se doutent bien de l'importance de ces questions et sont très attentifs aux conseils (« recettes » ou " gestes professionnels") entendus lors du stage de rentrée qu'ils effectuent en observateurs dans les classes des maîtres formateurs. Mais ils considèrent que, pour l'essentiel, la logique des apprentissages scolaires peut structurer la relation pédagogique. Pour eux, ce sont les didactiques des disciplines qui disciplinent.

\section{Deuxième période : la genèse du développement professionnel}

Au mois de décembre, lorsqu'ils rentrent de leur premier stage en responsabilité, les PE2 donnent deux explications aux "imprévus " qu'ils ont rencontrés au cours de leur pratique. D'une part, ils soulignent les écarts constatés entre ce qu'ils pensaient faire et ce qu'ils arrivaient réellement à faire durant leur stage. Ces écarts se traduisent par un sentiment permanent de manque de temps qu'ils expliquent par une surévaluation des capacités de leurs élèves qu'ils imaginaient plus autonomes qu'ils ne le sont en réalité. D'autre part, ils prennent conscience que leur action doit simultanément prendre en charge de multiples dimensions liées à la gestion du groupe et que leur représentation initiale était trop exclusivement orientée vers les apprentissages scolaires.

Bref, ils jugent qu'ils n'ont pas été suffisamment bien préparés. "Faire la classe » les oblige à des détours ou des adaptations qu'ils n'avaient pas anticipés. La posture de retrait qu'ils imaginaient n'a pas été possible dans les faits et ils ont fait l'expérience de la nécessité d'un engagement qui les expose et d'une relation pédagogique à laquelle ils ne s'étaient pas préparés et qu'ils ont du mal à nommer. Commence alors une nouvelle période qui s'étend jusqu'à la fin de l'année de PE2 et qui se poursuit sans doute l'année suivante. Les PE2 vont se débattre entre travail prescrit et travail réel. Leurs conceptions initiales, qui étaient proches, se diversifient, mais tous ont maintenant la possibilité d'expliciter leur conception initiale puisqu'elle se révèle en tension avec l'expérience de la responsabilité de la classe. Dans leurs propos, un mot revient très souvent : «idéal ». Les recommandations de l'IUFM sont jugées utopiques par rapport à leurs 
pratiques effectives. Ainsi, par exemple, un PE2 valorise la posture d'une débutante venue témoigner à l'IUFM :

«Elle prenait énormément de liberté par rapport au discours que nous on pouvait entendre, dont on est en train de se détacher petit à petit nous aussi... pas se contenter mais quelque part se dire qu'on ne pourra pas dans toutes les matières, la première année, être super efficace, donc il faudra choisir d'en laisser quelques-unes et se contenter de prendre des manuels ou des choses toutes faites, donc c'était assez drôle, parce que c'était quelque chose qui me perturbait et je vois qu'effectivement, il faut faire le deuil d'une certaine perfection et que c'est quelque chose de plutôt normal... on ne peut pas se prendre la tête pendant des heures et des heures, il faut faire avancer des choses, parce qu'il faut que le lendemain on soit efficace, qu'il y ait des apprentissages qui se fassent, que la classe elle tourne... donc c'était pas mal parce que je me suis reconnu dans ces questionnements... »

\section{Les similitudes entre les trajectoires des professeurs stagiaires}

\section{Première similitude : concevoir la notion de gestion de la classe}

Tout au long de l'année, les PE2 cherchent à élaborer une conception fonctionnelle de la gestion de la classe, ce qui n'est pas étonnant tant cette notion était absente de leur représentation initiale. Pour cela, ils traitent les écarts entre ce qu'ils pensaient faire et ce qu'ils arrivent à faire. Progressivement, ils appréhendent le déroulement de la journée scolaire comme un temps de vie partagé avec un groupe d'enfants qui n'est pas homogène, qui n'est pas d'humeur égale, qui n'est pas forcement intéressé par les activités scolaires et qu'il faut savoir " occuper ". Ils font de plus en plus référence à la notion de vie quotidienne du groupe classe, envisagé comme une entité dont ils doivent prendre soin.

Cette prise de conscience va modifier l'orientation de leurs préoccupations. Ils deviennent plus pragmatiques et évaluent la validité des conseils qu'ils reçoivent en fonction de leur nouvelle priorité qui est la classe. Ils intègrent alors la temporalité réelle du déroulement de la classe qui a son propre rythme et qui n'est plus uniquement scandée par la succession des activités prévues. Ils sentent la nécessité d'aménager des "petits moments » qu'il faut parfois savoir improviser pour varier le tempo de la journée et les actions des élèves. En fin d'année, tout en ayant conscience que l'improvisation n'est pas conforme à la tache prescrite, ils se sentent plus disponibles pour leurs élèves. Cela se traduit dans leurs propos par l'opérationnalisation du cahier-journal : celui-ci apparaît comme un outil pour faire classe, alors qu'en début d'année, seules les fiches de préparation semblaient jouer ce rôle. La plupart des PE2 trouvent des réponses dans l'action et n'expriment plus, lors des entretiens qui suivent les stages, les préoccupations qui paraissaient importantes dans les entretiens qui précédaient les stages.

\section{Deuxième similitude : leur conception des apprentissages scolaires évolue par le traitement des dilemmes}

Une partie des écarts à la prescription secondaire pose de véritables dilemmes aux PE2 : ce qu'ils souhaiteraient réaliser sur le plan didactique leur semble souvent incompatible avec la gestion de la classe. Aussi, pour faire face, révisent-ils les trois commandements de leur représentation initiale tournée vers les apprentissages des élèves :

- "il faut que les élèves soient actifs " : un commandement à réinterroger. L'idée qu'ils se faisaient de l'autonomie des élèves est mise à mal même si cette idée persiste tant qu'ils ne prennent pas en compte la situation d'enseignement telle qu'elle se présente. En effet, lors de leurs premières expériences de classe, ils ont tendance à s'en tenir à la représentation initiale qu'ils avaient de l'activité des élèves. Mais ils apprennent ensuite à faire confiance à leurs perceptions des situations dans lesquelles ils se débattent pour envisager des planifications plus adéquates. Une PE2, par exemple, explique que pendant le déroulement de sa séance, elle réalise qu'elle a préparé en postulant l'autonomie de ses élèves, sans tenir compte de son expérience lors des premiers jours de classe. Elle prend alors conscience qu'elle fait une séance irréaliste et que surtout, elle aurait pu se douter avant de commencer que cette séance ne fonctionnerait pas. À trop vouloir rendre ses élèves actifs, elle n'a pas su les occuper ;

- « il faut que les élèves parlent»: un commandement à relativiser. Les PE2 prennent rapidement conscience qu'il est impossible de traiter toutes les prises de parole des élèves. En fait, ils mesurent qu'ils ne peuvent prendre en compte que les interactions qui les intéressent, celles qui alimentent le déroulement de leur séance et qui vont donc dans le sens de la poursuite de leur objectif. La maîtrise de la gestion de la classe est à ce prix s'ils veulent avancer. Ils éprouvent alors un sentiment de malaise basé sur l'impression de manipuler les élèves pour les amener à exprimer 
les remarques attendues sans pouvoir, comme ils le souhaitaient, prendre réellement en compte la diversité de leurs interrogations comme l'exprime l'une d'entre eux :

"En fait du coup ce qu'il s'est passé par rapport à ce dont je me souviens, c'est qu'ils ont posé des questions ils ont émis des hypothèses et ensuite on est passé à autre chose en fait, je ne savais pas m'en servir en fait, je n'y arrivais pas, ça part dans tous les sens, et alors comment on les ramène ? Parce qu'on a un objectif notionnel derrière, parce qu'ils ont aussi des compétences à mettre en jeu et que ces hypothèses il faut qu'ils puissent aller les tester mais le problème c'est qu'on se retrouve avec des hypothèses et on n'a pas les moyens de les tester. Quant aux questions je n'en parle même pas parce que, parce qu'ils vont en poser 15000 et nous on peut pas toutes les traiter, et bien souvent on en a déjà une en tête d'ailleurs, c'est truqué en fait. »

À ce malaise s'ajoute la crainte de paraître directif et d'inhiber la parole des élèves. La gestion des interactions leur semble être un exercice d'équilibriste entre des postures d'ouverture qui permettent l'expression de chacun et des postures de fermeture qui ne retiennent que les remarques qui vont dans le « bon " sens : " il faut que l'élève parle, oui, mais pour faire avancer la leçon. ";

- "il faut développer l'autonomie des élèves": un commandement qui ne va pas de soi. Les PE2 mesurent que l'autonomie, souvent prônée, n'est pas une ressource pour faire classe. Ils découvrent que les élèves sont peu autonomes et qu'ils ne le deviennent pas naturellement sous prétexte que la situation proposée attend d'eux qu'ils le soient. Ils réalisent qu'ils doivent beaucoup encadrer l'activité des élèves pour que ces derniers puissent finir et réussir les tâches scolaires proposées.

\section{Troisième similitude : tenter d'assumer la fonction enseignante en étant exigeant sur le maintien des ressources attentionnelles des élèves}

Les PE2 ont du mal à assumer une fonction enseignante qui n'est pas conforme à leur personnalité. Certains prennent toutefois conscience de l'importance d'agir sur les attitudes des élèves. Ils comprennent que le maintien des ressources attentionnelles de ces derniers ne passe pas uniquement par l'élaboration de scénarios didactiques, mais aussi par une posture " encadrante " visant à réguler les comportements.

\section{Quatrième similitude : le recours au genre professionnel}

Les PE2 de notre cohorte découvrent une ressource précieuse : les autres enseignants qui, s'ils sont suffisamment semblables à eux ou attentifs à leur désarroi, peuvent potentiellement répondre à leurs interrogations. Ceux-ci, souvent, reconnaissent leurs préoccupations et leur proposent des pratiques alternatives qui se caractérisent par une prise en compte du réel quitte à modifier, voire à rejeter, la prescription secondaire. Cet ensemble de gestes partagés et de connivences sur ce qu'il est convenu de faire, désigné sous le terme de "genre professionnel » (Clot \& Faïta, 2001), apparaît à leurs yeux comme une référence stable et commune qui peut leur assurer une protection et un soutien face à la solitude des décisions qu'ils ont à prendre pour faire classe. Les PE2 s'en emparent pour assumer la tension née des écarts et des dilemmes auxquels ils sont soumis durant leurs stages. Phénomène intéressant, nombreux sont ceux qui expliquent qu'ils ont dû en partie renoncer à la prescription du centre de formation pour faire classe, mais qu'ils ont fait en sorte que ce choix ne se remarque pas les jours de visite de leurs conseillers/ évaluateurs.

Ces similitudes dans le processus de redéfinition de la tâche ne seront pas appréhendées de la même manière par tous les PE2. D'autant plus que des conditions du dispositif de formation peuvent les influencer et jouer un rôle déterminant sur l'évolution de leur parcours.

\section{Les déterminants de ce processus}

\section{Première condition : préserver un sentiment de réussite lors des stages}

Lorsqu'on demande à V., une PE2 qui prend le risque d'affronter les situations d'enseignement, ce qui lui donne le droit de rejeter, de modifier ou d'adapter une prescription, elle évoque le sentiment d'avoir suffisamment confiance en son propre travail de préparation. Lorsqu'on lui demande d'où vient cette confiance, elle fait référence aux entretiens avec son équipe de tutorat qui a pris en compte l'état de sa réflexion.

D'une manière plus générale, les PE2 plébiscitent certaines caractéristiques des entretiens qui favorisent, à leurs yeux, une mise en confiance. Ils évoquent les conseils des formateurs qui cherchent à comprendre leurs raisons d'agir et qui s'appuient sur leurs difficultés à faire la classe pour émettre des 
conseils plutôt que d'insister sur le respect des normes du « didactiquement correct ". Par exemple, les PE2 soulignent l'intérêt des entretiens avec leurs tuteurs qui démarrent par une clarification des intentions qu'ils avaient lors du temps de préparation. Ils préfèrent ces tuteurs à ceux qui proposent des séances alternatives plus performantes, mais qui ne prennent pas en compte l'état initial de leurs préoccupations. Les PE2 se sentent en réussite lorsqu'ils sont en mesure de comparer ce qu'ils savent faire avec ce qu'ils ne savent pas encore faire et surtout de pouvoir refaire seuls ce qu'on leur a conseillé de faire. C. le revendique :

"Là, elle [la formatrice] me donne un truc, ce que j'apprécie d'ailleurs, par rapport à l'autre visite où en voulant répondre à mes problèmes on m'a changé complètement ma séance, ça n'avait plus rien à voir avec ce que j'avais à l'origine, dans ce cas-là ça me parle beaucoup moins quoi, moi je préfère ce genre de conseils, je préfère des conseils qui restent proches de ce que j'ai fait, et que ça me parle du coup. "

Maintenir sa capacité d'agir semble donc nécessaire pour s'engager sur la voie de la conception de sa propre tâche, face au risque destructeur du sentiment d'échec général qui se traduit par l'impression d'être submergé et de ne plus pouvoir faire face à rien.

\section{Deuxième condition : permettre au PE2 de conserver suffisamment d'estime de soi pour construire une identité professionnelle}

L'exposition aux regards des autres rend les PE2 très sensibles aux remarques qui touchent leurs attitudes face aux élèves. Ils réagissent vivement, par exemple, à la manière dont un formateur les reprend sur une erreur de langage ou d'orthographe laissée au tableau. Ils se sentent atteints dans l'estime qu'ils ont d'eux-mêmes et semblent déstabilisés dans leur engagement à prendre en charge la classe. Les remarques qui portent sur leur manière d'être ou de parler sont souvent jugées agressives. En outre, les conseils les invitant à théâtraliser leur comportement sont également mal perçus car ils font référence à un jeu qui révèle une intention masquée, voire une volonté de tromper. Cette interprétation les conduit à rejeter des conseils qu'ils entendent comme des invitations à duper les élèves, d'autant plus que leur intention initiale d'enseignement est entièrement basée sur la sincérité de leur motivation. Les interpellations sur leur comportement fragilisent la construction d'une identité professionnelle et peuvent les conduire à inhiber leur engagement pour adopter une posture plus attentiste ou à refuser la formation par une attitude revendicative, voire provocatrice.
Troisième condition : présenter des outils qui aident à faire classe et à faire apprendre

Les PE2 évoquent des outils didactiques comme des outils organisationnels dont l'expérimentation, lors des stages, les a aidés à se sentir plus efficaces. Ils font alors référence aux présentations qui leur ont été faites en cours. La logique d'action qui prime lors de ces présentations rend plus facile l'expérimentation lors des stages. Les manuels sont rarement présentés dans cette optique. Le plus souvent, les formateurs disciplinaires les présentent dans le but d'identifier et d'analyser les choix didactiques qui président aux contenus. Cette finalité ne permet pas aux PE2 d'identifier les manuels comme des outils pour faire la classe au quotidien. Bien au contraire, les critiques émises lors des analyses les conduisent à être très circonspects sur leur usage. Ils renoncent donc le plus souvent à s'en servir, convaincus que ces outils ne sont pas à la hauteur des exigences des formateurs de I'IUFM. Mais ils se trouvent fort démunis lorsqu'arrive l'épreuve de la classe.

\section{Des profils d'évolution des PE2}

Les PE2 réagissent différemment aux situations d'apprentissages qu'ils rencontrent durant l'année. Toutefois, les régularités que nous avons repérées grâce à l'analyse de notre corpus nous permettent de constituer trois profils capables de rendre compte des évolutions singulières de leur représentation initiale :

- premier profil (rare) : l'abandon quasi complet de la représentation initiale, le renoncement à l'idéal du centre de formation. Dans ce cas, c'est souvent la remise en cause de la formation qui domine dans les propos des PE2. La coupure entre ce qu'ils appellent " la théorie » et « la pratique » est nette. Ils pensent que " la théorie » est une utopie et que la formation n'arrivera pas à régler les problèmes qu'ils rencontrent en classe. Pour eux, cet idéal est une vue de l'esprit. Dans le meilleur des cas, ils se tournent vers leurs collègues pour rechercher des solutions qui, à leurs yeux, sont plus pragmatiques. Ce premier profil correspond à des stagiaires qui disent que l'IUFM est à côté de la réalité et que, s'ils veulent apprendre leur métier, il vaut mieux qu'ils s'adressent à leurs collègues, dans les écoles.

- deuxième profil (rare) : le maintien de la représentation initiale, l'affirmation d'un idéal. Cette posture montre un attachement aux conceptions véhiculées par la prescription secondaire. Les PE2 vivent l'expérience de la classe comme un appel au renoncement d'un idéal qui les a portés pendant tout le 
temps des épreuves du concours (PE1). Ils refusent les compromis que nécessite la prise en compte des situations d'enseignement dans lesquelles ils se débattent. Ils ajournent leur volonté de réussite pédagogique et considèrent qu'ils n'ont pas les moyens durant les stages de réaliser une pratique de classe conforme à leur représentation de la tâche d'enseignement. Ce deuxième profil correspond plus à des stagiaires qui explicitent très clairement les ajustements qu'ils devraient faire, mais n'arrivent pas à s'engager dans l'action. Ils ne se sentent pas prêts, car ils jugent qu'ils manquent de connaissances didactiques. Cette conception leur permet de garder intact leur idéal.

- troisième profil (très majoritaire) : l'engagement dans un processus de redéfinition de la tâche, un idéal instrumenté. Cette posture se caractérise par une préoccupation nouvelle pour la réussite du stage. Celui-ci devient le principal catalyseur de la formation. Les PE2 engagés dans cette voie sélectionnent les conseils qu'ils retiennent en fonction de l'aide qu'ils pensent pouvoir en retirer durant les stages. La pratique devient le but et le moyen de leur formation. Ils prennent progressivement conscience qu'ils ne peuvent se contenter de tenter d'appliquer ce qu'ils ont compris de la prescription, mais qu'ils doivent transposer ce qu'ils apprennent pour en faire des règles d'action capables de les aider à enseigner. Le modèle idéal de l'IUFM devient alors un repère qui les aide à prendre des risques, à s'engager sur le chemin de l'élaboration de leur propre pratique. Le PE2 prototype de ce troisième profil s'engage dans une action réfléchie, mais proteste souvent, car il a le sentiment qu'on ne lui dit pas tout et qu'il devra s'en sortir par lui-même. II apprend à trouver des ressources et des soutiens dans le dispositif de formation, suivant l'actualité de ses préoccupations professionnelles.

\section{CONCLUSION}

Au terme de cette étude, il apparaît que les représentations fonctionnelles initiales, construites avant tout passage à l'action, jouent un rôle décisif dans le développement professionnel. Dès le début de l'année, les professeurs stagiaires disposent d'une représentation du métier forgée avant la réussite au concours. Celle-ci n'est pas spécifique à un contenu didactique particulier ; elle regroupe des principes pédagogiques généraux, peu contextualisés, qui doivent guider l'action des professeurs stagiaires ; elle est essentiellement élaborée à partir des savoirs scientifiques délivrés en formation sur la manière dont les élèves apprennent. Comme on pouvait s'y attendre, elle s'avère peu opérante lors du premier stage en responsabilité.

Cette représentation initiale, largement partagée par les stagiaires et que l'on peut qualifier de " générique » au sens où elle ne tient pas compte de la spécificité des contenus didactiques, s'est construite durant la préparation au CRPE (Concours de recrutement des professeurs des écoles). Tous ont quasiment la même représentation de la classe : une classe active dans laquelle les élèves " construisent leurs savoirs", en référence à la démarche pédagogique basée sur le modèle socioconstructiviste. Ils adhèrent d'autant plus à ces principes que ce qu'ils ont appris pendant l'année de PE1 a été validé par leur propre réussite au concours, y compris lors de l'entretien professionnel présidé par un inspecteur de l'Éducation nationale. Les professeurs stagiaires ne soupçonnent pas de différences entre les attentes de l'IUFM et celles de leur employeur : pour eux, prescriptions primaire et secondaire ne font qu'une. Ils pensent avoir bien compris ce qu'on attend d'eux et ils jugent cette attente légitime. Ils y trouvent une motivation qui donne du sens à leur engagement professionnel. Ils construisent alors une représentation initiale qui modifie celle antérieurement acquise dans d'autres situations vécues de manière singulière par chaque stagiaire (formation BAFA, souvenirs d'école, stage préprofessionnel fait durant la licence...). Cette représentation partagée leur permet d'interpréter les situations d'enseignement auxquelles ils assistent lors des premiers stages d'observation. Lorsque celles-ci ne correspondent pas à la représentation qu'ils ont construite, ils critiquent ce qu'ils voient dans les classes et n'imaginent pas abandonner leur représentation initiale. II faudra attendre l'expérience du premier stage en responsabilité pour qu'ils entrevoient les raisons de la modifier afin de la rendre plus opérationnelle.

Le premier stage en responsabilité malmène sérieusement les représentations initiales, car il active une multitude de préoccupations que les débutants n'arrivent pas à gérer, d'une part parce qu'il faudrait les traiter de manière simultanée (d'où le sentiment de devoir tout accélérer) et d'autre part parce qu'ils n'arrivent pas à établir un ordre de priorité entre elles (d'où le sentiment de frustration face aux choix à effectuer). Ce que les stagiaires jugeaient bon de faire leur semble créer plus de problèmes de conduite de classe que de solutions. Souvent confrontés à des situations qu'ils n'avaient pu ni anticiper ni se représenter, les débutants remettent en cause la 
prescription du centre de formation, suspectée de faire référence à des situations d'enseignement idéales et irréalisables pour des débutants :

" Alors voilà il faut qu'ils [les élèves] se posent des questions, à partir de ces questions il faut qu'ils émettent des hypothèses, et qu'ils soient eux-mêmes chercheurs et eux-mêmes actifs dans leur apprentissage, très bien, sauf que, quand c'est moi, je dois les amener là, je n'ai pas le choix, donc soit je leur donne, soit et c'est ce que j'essaie plus ou moins de faire, j'ai trouvé ça comme solution, c'est-à-dire les amener à se poser des questions à émettre des hypothèses mais c'est truqué quoi, c'est-à-dire que moi je me débrouille pour qu'ils se posent cette question, même si ce n'est pas fait avec grande classe. ”

Ils cherchent alors à élaborer une nouvelle représentation fonctionnelle pour conduire leur classe dans le plus grand nombre de situations possibles. Celle-ci apparaît comme la conséquence de l'impossibilité à mettre en œuvre la représentation initiale jugée inaccessible à des débutants. Cela leur permet d'expliquer et d'excuser leurs difficultés en stage. D'autant plus que les pratiques observées durant leur formation (en PE1 et en début de PE2) sont celles de maîtres expérimentés, la plupart du temps des professeurs des écoles maîtres formateurs. II leur semble donc normal de ne pas parvenir à les mettre en œuvre.

Dès lors, cet idéal devient, dans le meilleur des cas, un point de repère qui oriente leurs efforts et leur réflexion, un horizon vers lequel tendre progressivement ; dans le pire des cas, il sert de repoussoir. On comprend ainsi pourquoi certains professeurs stagiaires rejettent en bloc les prescriptions du centre de formation et renoncent à l'idéal pédagogique qu'il promeut. D'autres, refusant les compromis qu'implique la pratique, en restent à l'idéal et ajournent leur passage à l'acte pédagogique : ils tergiversent et ne s'engagent pas vraiment dans le travail. Ils seront les premiers à accepter un poste dans l'enseignement spécialisé... ou à s'inscrire en doctorat!

Mais, dans la majorité des cas, les professeurs débutants, lorsqu'ils sont rassurés sur leur capacité à " rester maîtres de la situation " face aux dilemmes rencontrés, s'engagent dans une activité de " traitement » de leurs premières expériences en les soumettant aux filtres de la prescription générique idéale. Ils essaient alors de rapprocher les situations parfois difficiles qu'ils ont vécues et les situations étudiées en cours. Ils s'efforcent d'isoler et d'identifier des principes d'actions capables de leur être utiles dans d'autres situations d'enseignement. Ils arrivent ainsi à construire progressivement une représentation plus opératoire de la tâche d'enseignement, à condition que leurs premières expériences, accompagnées par des formateurs compétents, soient sources de réussites et non synonymes d'échecs.

Nos résultats nous autorisent donc à remettre en cause les scénarios de formation qui ont, pour les débutants, des exigences trop élevées, par exemple ceux qui leur demandent d'exploiter les erreurs de leurs élèves avant de leur avoir appris à canaliser l'attention de ceux-ci lors des phases d'exposition des notions. Ils nous permettent aussi de mieux comprendre les critiques adressées par les stagiaires à leurs formateurs comme le résultat d'un excès d'ambition. Autrement dit, si l'on attend trop et trop tôt d'un débutant, on court le risque de le renvoyer à une impuissance résolument démobilisatrice.

Marc Daguzon marc.daguzon@univ-bpclermont.fr Université Blaise-Pascal, Laboratoire ACTé

Roland Goigoux roland.goigoux@univ-bpclermont.fr Université Blaise-Pascal, Laboratoire ACTé 


\section{BIBLIOGRAPHIE}

ALTET M. (1994). La formation professionnelle des enseignants. Paris : PUF.

BAILLAUQUÈS S. (1999). “ Ce que l'entrée dans la carrière révèle du rapport des enseignants à la formation : éléments d'une problématique de professionnalisation ». In J.-C. Hétu, M. Lavoie \& S. Baillauquès (dir.), Jeunes enseignants et insertion professionnelle. Un processus de socialisation ? De professionnalisation ? De transformation ? Bruxelles : De Boeck, p. 21-41.

BLANCHARD-LAVILLE C. \& NADOT S. (dir.) (2000). Malaise dans la formation des enseignants. Paris : L'Harmattan

BUTLEN D., PELTIER-BARBIER M.-L. \& PÉZARD M. (2002). "Nommés en REP, comment font-ils ? Pratiques de professeurs des écoles enseignant les mathématiques en REP : contradictions et cohérence ». Revue française de pédagogie, no 140, p. 41-52.

BUTLEN D. (2004). Apprentissages mathématiques à l'école élémentaire. Des difficultés des élèves de milieux populaires aux stratégies de formation des professeurs des écoles. Habilitation à diriger des recherches (HDR), sciences de l'éducation, université Paris 8.

CARTER K. \& DOYLE W. (1987). “ Teachers' knowledge structures and comprehension processes ". In J. Calderhead (dir.) Exploring teachers' thinking. Londres : Cassel, p. 147-160.

CLOT Y. \& FAÏTA D. (2001). " Genre et style en analyse du travail. Concepts et méthodes ». Travailler, no 6, p. 7-42.

DAGUZON M. (2009). « De la prescription à la redéfinition de la tâche d'enseignement (étude longitudinale) ". In R. Goigoux, L. Ria \& M.-C. Toczek-Capelle (dir.), Les parcours de formation des enseignants débutants. Clermont-Ferrand : Presses universitaires BlaisePascal, p. 101-120.

DAGUZON M. (2010). L'influence de la prescription sur le développement professionnel des professeurs des écoles débutants. Thèse de doctorat, sciences de l'éducation, université Blaise-Pascal.

DUBAR C. (2002). La socialisation : construction des identités sociales et professionnelles. Paris : Armand Colin.

DUBET F. (2002). Le déclin de l'institution. Paris : Éd. du Seuil.

FREESE A. R. (1999). "The Role of Reflection on Preservice Teachers' Development in the Context of a Professional Development School ». Teaching and Teacher Education, no 15, p. 895-909.

GOIGOUX R. (2002). " L'évolution de la prescription adressée aux instituteurs : l'exemple de l'enseignement de la lecture entre 1972 et 2002 ». In J.-M. Evesque, A.-M. Gautier, C. Revest \& Y. Schwartz (dir.), Les évolutions de la prescription. Actes du XXXVII congrès de la Société d'ergonomie de langue française. Aix-enProvence : GREACT, p. 77-84.

GOIGOUX R. (2006). "Ressources et contraintes dans le travail d'enseignement de la lecture au cours préparatoire ». In B. Schneuwly \& T. Thévenaz (dir.), Le travail de l'enseignant et l'objet enseigné : le cas du français langue première. Bruxelles : De Boeck et Lancier, p. 67-92.

GOIGOUX R. (2007). «Un modèle d'analyse de l'activité des enseignants ". Éducation et didactique, n० 3 , p. $19-41$.

GOIGOUX R. \& PARIES M. (2006). «Réduire la complexité de l'activité d'enseignement pour mieux préparer les professeurs débutants à l'action et à la réflexion ". In Actes du colloque international Formation d'enseignants. Quels scénarios? Quelles évaluations? En ligne : http://www.versailles.iufm.fr/colloques/ scenarios/pdf/synth_gr4_07.pdf (consulté le 1er mars 2007)

GOIGOUX R., RIA L. \& TOCZEK-CAPELLE M.-C. (dir.) (2009a). Les parcours de formation des enseignants débutants. Clermont-Ferrand: Presses universitaires Blaise-Pascal.

GOIGOUX R., RIA L. \& TOCZEK-CAPELLE M.-C. (2009b). "Mieux connaître les parcours de formation des enseignants débutants pour mieux les former ». In R. Goigoux, L. Ria \& M.-C. Toczek-Capelle (dir.), Les parcours de formation des enseignants débutants. Clermont-Ferrand : Presses universitaires BlaisePascal, p. 25-44.

HARRINGTON H. L., QUINN-LEERING K. \& HODSON L. (1996). «Written case analyses and critical reflection ». Teaching and Teacher Education, n² 2, p. 25-37.

HUBERMAN M. (1989). La vie des enseignants. Évolution et bilan d'une profession. Lausanne et Paris : Delachaux et Niestlé.

KAGAN D. M. (1992). «Professionnal growth among preservice and beginning teachers ". Review of Educational Research, no 62, p. 129-169.

LANG V. (1999). La professionnalisation des enseignants. Paris : PUF.

LEGENDRE M.-F. (1998). « Apport du modèle de l'équilibration à l'étude de l'apprentissage chez l'adulte ». In C. Danis \& C. Solar (dir.), Apprentissages et développement des adultes. Montréal : Les Éditions Logiques, p. 167-232.

LEPLAT J. (1985). Erreur humaine, fiabilité humaine dans le travail. Paris : Armand Colin.

LEPLAT J. (1992). " Les représentations fonctionnelles dans le travail ». In J. Leplat (dir.), L'analyse du travail en psychologie ergonomique. Toulouse : Octarès, p. 107-120.

LEPLAT J. (1997). Regards sur l'activité en situation de travail. Paris : PUF.

LEPLAT J. \& HOC J.-M. (1983). «Tâche et activité dans l'analyse psychologique des situations ». Cahiers de psychologie cognitive, $\mathrm{n}^{\circ} 3$, p. 49-63.

MAULINI O. (2009). "Devenir enseignant : invariants et évolution des débuts dans la profession ». In R. Goigoux, L. Ria \& M.-C. Toczek-Capelle (dir.), Les parcours de formation des enseignants débutants. Clermont-Ferrand : Presses universitaires BlaisePascal, p. 61-78. 
OCHANINE D. (1978). "Le rôle des images opératives dans la régulation des activités de travail ». Psychologie \& éducation, n०3, p. 63-65.

PAQUAY L., ALTET M., CHARLIER E. \& PERRENOUD P. (dir.) (2012). Former des enseignants professionnels. Quelles stratégies? Quelles compétences? Bruxelles: De Boeck.

PASTRÉ P. (2005). « Genèse et identité ». In P. Rabardel \& P. Pastré (dir.), Modèles du sujet pour la conception. Dialectiques activités développement. Toulouse : Octarès, p. 231-260.

PASTRÉ P. (2008). « La didactique professionnelle : origines, fondement, perspectives ». Travail et apprentissages, no 1, p. 9-21.

PASTRÉ P. (2011). La didactique professionnelle. Paris : PUF.

PELTIER-BARBIER M.-L. (2004). Dur, dur d'enseigner en ZEP. Grenoble : La Pensée sauvage.

PIOT T. (1997). " Les représentations des enseignants débutants sur leurs pratiques ». Recherche et formation, $n^{\circ} 25$, p. 113-123.
ROBERT A., GOIGOUX R., BRIGAUDIOT M. et al. (2006) Formation d'enseignants: quels scénarios? Quelles évaluations ? Colloque organisé les 16 et 17 mars 2006 à Antony. En ligne : http://www.versailles.iufm.fr/ colloques/scenarios/ (consulté le 1er mars 2007).

ROGALSKI J. \& LEPLAT J. (2011). « L'expérience professionnelle : expériences sédimentées et expériences épisodiques ". @ctivités, vol. 8, n². En ligne: http:// www.activites.org/v8n2/rogalski.pdf (consulté le 24 avril 2009).

TARDIF M. \& LESSARD C. (1999). Le travail enseignant au quotidien. Expérience, interactions humaines et dilemmes professionnels. Paris : De Boeck.

TILLEMA H. H. (2000). "Belief change toward self-directed learning in student teachers : immersion in practice or reflection on action ". Teaching and Teacher Education, n० 165, p. 75-591.

WITTORSKI R. (2007). Professionnalisation et développement professionnel. Paris : L'Harmattan.

WITTORSKI R. \& BRIQUET-DUHAZE S. (2008). Comment les enseignants apprennent-ils leur métier ? Paris : L'Harmattan. 